\title{
LE THÈME DE LA RÉVOLUTION DANS LA PENSÉE DE SARTRE*
}

\author{
Cristina Diniz Mendonça CREMA**
}

RÉSUME: L'oeuvre de Sartre pourrait être lue comme l'incarnation de l'es prit "pathétique" et "héroïque" de l'époque de la guerre et de la Résistance. Sous le coup de feu des barricades de 44, une philosophie de la révolution commence à éclore-elle fut forgée à chaud durant cette époque de "haute température historique". C'est de la généralisation théorique de cette expérience politique cruciale, c'est-à-dire de la cristallisation du "mythe" de la Résistance, que vient, à mon avis, l'idée satrienne de révolution.

UNITERMES: Résistance; révolution; apocalypse; socialisme; liberté.

Après la défaite française de 1940, un historien allemand, représentant de l'historiographie officielle du national-socialisme, écrit la chose suivante: "Nous avons atteint deux objectifs: nous avons détruit la ligne Maginot et nous avons extirpé 1789 du coeur des hommes".

Si 1940 a pu signifier l'éclipse de l'esprit de 1789, 1944 a signifié pour l'imaginaire de l'époque - et très particulièrement pour Sartre - justement la renaissance de cet esprit. Avec la Libération, c'est toute la tradition révolutionnaire française qui est célébrée en même temps.

C'est à l'ombre de cette célébration de la tradition révolutionnaire française que la pensée de Sartre prit forme. Sa conception de l'histoire et de la révolution semble avoir fleuri pendant cette époque drapée dans la brume de l'enthousiasme révolutionnaire. Emporté par la "force des choses", pour utiliser l'expression consacrée par Saint-Just et

* Ce texte a été présenté comme communication au Colloque du Groupe d'Etudes Sartriennes, juin 1989, à Paris (Sorbonne).

** L'auteur est prof esseur à l'Université de l'Etat de São Paulo. Depto. de Filosofia da Faculdade de Filosofia e Ciências-UNESP-17500-Marília-SP. 
reprise par Simone de Beauvoir, Sartre, sans tradition dialectique, est tiré vers l'histoire, la Révolution, le marxisme. "Intellectuels modérés" - dit-il dans "Merleau-Ponty vivant" - la Résistance nous avait tirés à gauche". C'est la "proximité imaginaire" de la révolution, ou même sa proximité réelle, qui conduit Sartre à la découverte de la théorie de la révolution. La pensée sartrienne, son idéal du concret et de la totalité, son apologie de la Révolution, pourrait ainsi être considérée comme le résultat théorique d'un moment historique privilégié où il était possible de saisir le social comme un tout. Bref, c'est dans le sol historique de la France de l'Occupation, de la Résistance et de la Libération que la philosophie de Sartre se trouve enracinée.

Il est bien vrai, certes, que l'on peut déjà entrevoir en 34, dans La Transcendance de l'ego, une esquisse du projet philosophique sartrien. Mais la forme particulière de son développement n'aurait été possible qu'à partir de l'expérience politique de la guerre et de la Résistance. Ce n'est pas par hasard que L'Etre et le néant est sorti des Carnets de la drôle de guerre. Ce livre pourrait se lire comme une transposition philosophique de cette expérience historique vécue. Laissorıs Sartre lui-même se prononcer sur Les Carnets de la drôle de guerre: "Je le considère comme un témoignage: le témoignage d'un bourgeois de 1939 mobilisé, sur la guerre qu'on lui fait faire. (...) la valeur historique de mon témoignage me justifie à le faire." (p. 90)

$\mathrm{Si}$, d'un côté, la découverte de la phénoménologie allemande a été assez décisive pour que l'on puisse dire que la pensée de Sartre ne naît vraiment qu'après cette découverte, de l'autre côté, c'a éte l'expérience de la guerre et de la Résistance qui a donné définitivement forme à cette pensée qui alors à peine s'esquissait. Le vrai visage de la philosophie sartrienne ne peut pas être dégagé avant cette expérience historique. Ou mieux: la "découverte" de Heidegger en elle-même est déjà, d'après le bilan de Sartre, une tentative de réponse théorique aux problèmes posés par l'histoire face à la menace imminente de la guerre. A cette époque où l'Histoire, avec un $\mathrm{H}$ majuscule, imposait sa présence aux contemporains, comme l'indiquent Les Carnets de la drôle de guerre, il fallait une philosophie "pathétique" et "héroïque" telle que Sartre définit la philosophie heideggerienne. La "découverte" de cette philosophie est donc une anticipation des problèmes théoriques posés par un temps considéré, lui aussi, "pathétique" et "héroïque". Comme Balzac, Sartre anticipe, au niveau des idées, les transformations sociales de son époque. C'est ainsi que, à partir de l'esprit de la guerre et de la Résistance, Sein und Zeit a pu renaître à travers L'Etre et le néant.

On sait que pour Hegel, comme l'a souligné Habermas (dans "Les Ecrits Politiques de Hegel"), "la guerre est le moyen par lequel l'histoire universelle étend son destin sur les peuples". Que l'on se souvienne du célèbre passage de "Questions de Méthode": "C'est la guerre qui fit éclater les cadres vieillis de notre pensée. La guerre, l'occupation, la Résistance, les années qui suivirent. "Le résultat de cet éclatement fut l'épanouissement de la pensée sartrienne.

Mais où est-ce que je veux en venir? Ce que je voudrais montrer, c'est que la philosophie sartrienne de la Révolution émane de la Résistance, ou plus précisément qu'elle fut forgée 
à chaud dans la lutte politique pour la Libération. Bien que Sartre n'ait pas participé directement à cette lutte-sauf pendant la brève expérience du groupe "Socialisme et Liberté" - , ce qui importe, c'est qu'il a élaboré théoriquement ce que les autres, les Résistants, ont fait dans la pratique politique (pratique dont il fut témoin). L'oeuvre de Sartre elle-même est, beaucoup plus que l'on ne pourrait le penser de prime abord, l'expression, ou l'illustration, d'une conjoncture historique de crise révolutionnaire, c'est-à-dire la lutte pour la libération de la France occupée. Plusieurs concepts sartriens fondamentaux sont nés, à mon avis, au moment des barricades de 44. Dans cette perspective, l'expérience politique de 44 aurait eu sur la pensée de Sartre une valeur structurelle, pas seulement conjoncturelle. En ce sens, on pourrait considérer l'oeuvre sartrienne comme une sorte de témoignage (philosophique et litéraire) de l'expérience politique de la guerre et de la Résistance (un témoignage qui a une "valeur historique", comme le disait Sartre au sujet de ses Carnets).

Si cette idée est exacte, il y aurait une expérience historique réelle à l'intérieur du mouvement spéculatif des concepts sartriens. Ces concepts, malgré leur apparence abstraite, auraient des racines dans l'expérience historique concrète. La philosophie sartrienne pourrait ainsi être lue comme l'incarnation de l'esprit "pathétique" et "héroïque" de cette époque de la guerre et de la Résistance. Ou mieux, elle serait la narration - une narration épique-de cette expérience "apocalyptique" de la Résistance. C'est de la généralisation théorique de cette expérience, c'est-à-dire de la cristallisation du "mythe" de la Résistance, que vient, à mon avis, l'idée sartrienne de Révolution. C'est cela que je vais essayer de développer.

"De la Résistance à la Révolution" - voilà le mot d'ordre qui, pendant la "fête" de la Libération, selon l'expression de Sartre, rassemblait les intellectuels qui venaient de sortir du combat contre le nazisme. Dans cette conjoncture d'effervescence révolutionnaire de l'après-guerre français, c'étaient pour ainsi dire dans l'air, les principaux thèmes qui deviendront partie intégrante de la pensée sartrienne: le lien entre morale et politique, socialisme et liberté, Résistance et Révolution, les thèmes de l'héroïsme, de l'engagement et de l'humanisme. Ces idées font partie de l'esprit de ce temps-du Zeitgeist. C'est cette expérience historique, fondamentale qui se trouve à l'origine de la philosophie de Sartre.

Si l'on jette un coup d'oeil sur la presse de l'époque (soit la presse clandestine pendant l'occupation, soit la presse des jours de la Libération), on peut comprendre que les thèmes de l'héroïsme et de l'engagement naissent (ou renaissent) ensemble durant la guerre, l'Occupation et la Résistance (Note 1).

Le journal Combat (dirigé par Camus et dans lequel Sartre écrivait) en est un bon exemple. Portant en sous-titre le mot d'ordre "De la Résistance à la Révolution", Combat, en 44, fait appel à un "effort en commun" et à l'héroïsme de tous pour que la tâche de la Résistance ne finisse pas avec la Libération. Elle devrait continuer jusqu’à ce qu'une Révolution réalise vraiment la liberté."Ayant commencé par la Résistance-dit un éditorial 
de ce journal - finir par la Révolution". Et dans un éditorial postérieur, on lit: "Ce que nous appelons une révolution, c'est supprimer la politique pour la remplacer par la morale. "Cette révolution-ajoute le journal-pourrait se faire "dans l'ordre et dans le calme", c'est-à-dire sans violence.

Evidemment, Sartre n'adoptera jamais cette idée de révolution (dans laquelle on peut déjà reconnaître les traits du "libéralisme" de Camus critiqués plus tard par Sartre lui-même). Cependant, les éditoriaux du Combat viennent confirmer l'idée que le lien entre politique et morale faisait partie de l'esprit de l'époque. Et ce lien sera toujours présent dans la pensée sartrienne. Déjà en 1939, dans Les Carnets de la drôle de guerre, Sartre, citant Mauriac, dit que "la question éternelle qui a toujours divisé les Français (...) touche aux rapports de la politique et de la morale" (p. 121). Avec la guerre, cette "question éternelle" française prenda bien sûr des couleurs nouvelles et plus vives.

Il convient de rappeler que ce problème des rapports entre la politique et la morale avait déjà été très vivement à l'ordre du jour pendant la période de la Révolution Française. Comme on le sait, Robespierre et les membres des clubs révolutionnaires soumettaient la politique à la morale. Sartre, dans un manuscrit inédit sur la Révolution Française (écrit probablement vers 56 - il s'agit d'un scénario de film ayant comme personnage principal le conventionnel Joseph LeBon) (Note 2), indique que le lien établi par les jacobins entre homme et citoyen renvoie aux rapports entre morale et politique. On va voir que c'est justement cet esprit de 89 qui renaît pendant la période de la Résistance.

Selon Combat, "l'esprit révolutionnaire issu de la Résistance" est celui capable d'unir une "politique de liberté" à une "politique d'honneur". "Il vient un temps-proclame le journal-où la morale rentre dans la politique". C'est pendant cette "époque pleine de sacrifices et de grandeurs secrètes", pour me servir encore d'une expression du Combat, que la notion d'engagement naît comme une catégorie à la fois politique et morale.

Ce "temps de la morale" est aussi celui de la liberté héroïque ou de l'héroïsme libertaire. C'est ainsi que les éditoriaux des Lettres Françaises (clandestines)-organe du comité national des écrivains et auquel Sartre collaborait-ne cessent d'exalter le courage et l'héroïsme des martyrs de la lutte pour "la liberté renaissante". Rappelons-nous encore que Merleau-Ponty a décrit la Résistance comme une aventure héroïque de l'individu dans l'histoire: "Les Résistants ne sont ni des fous ni des savants, ce sont des héros" (Humanisme et Terreur). Et dans le numéro inaugural des Temps Modernes, la Résistance fut saluée comme l'expérience du "bonheur au milieu du danger." ("La guerre a eu lieu"). L'incorporation théorique du thème de l'héroïsme est un tribut que toute une "génération intellectuelle" paie à la Résistance.

Ces années de l'Occupation ont été définies par Combat comme "les années de la fraternité virile". D'ailleurs, ce thème de la fraternité virile (ou de l'héroïsme) revient sans cesse chez Malraux. Pour lui, comme Caute l'a souligné(Le Communisme et les Intellectuels Français), "la révolution était le véhicule de l'action par laquelle l'homme est conf ronté avec sa destinée tragique: la mort". Que l'on se souvienne, par exemple, de ce passage de Pour 
Thaelmann: "Cet homme qui disait qu'il venait à nous parce qu'il voulait la fraternité virile, cette fraternité qu'il avait cherchée sans la trouver dans la guerre, qu'il attendait de la révolte, et qu'il eût trouvée dans la Révolution (...)". Ces aspects romantiques et héroïques de l'esprit révolutionnaire, présents chez Malraux, exerceront une influence décisive sur Sartre ("je fais époque avec Malraux" - dit-il dans Les Carnets de la drôle de guerre). Pour qui était "pénétré par un idéal de vie emprunté au romantisme", comme on peut le lire encore dans Les Carnets de la drôle de guerre, l'esprit de l'époque était très favorable.

"Toute la ville aux Barricades." "Ils ne passeront pas." Avec ces mots d'ordre enflammés, le journal Combat évoque, au moment de la Libération de Paris, l'esprit de la guerre civile espagnole. Ce n'est pas par hasard, comme on va le voir, que Sartre, pour penser la Révolution, emprunte l'idée d'Apocalypse au roman de Malraux sur la guerre civile (L'Espoir). En outre, Sartre lisait, pendant la "drôle de guerre", Le Testament espagnol d'Arthur Koestler. Et il relève un passage où Koestler, décrivant quelques moments hérö̈ques où "nous surmontions même la peur de mourir", exalte la liberté de ces moments: "Dans ces heures-là, nous étions libres (...); c'était l'expérience de la liberté la plus absolue qu'un homme puisse connaître" (pp. 86-7). Or, c'est justement comme un moment privilégié de liberté que Sartre plus tard va décrire l'action héroïque de la Résistance: "Jamais nous n'avons été plus libres que sous l'Occupation allemande. (...) La Résistance a été le dévoilement même de notre liberté" ("La République du silence", 1944). Il semble donc qu'à l'origine de ces réflexions sartriennes se trouve, outre l'idée héroïque et romantique de la Révolution présente chez Malraux, le livre de Koestler sur l'Espagne.

Mais, plutôt qu'une reprise de l'esprit de la guerre civile espagnole, 1944 a signifié pour l'imaginaire de l'époque, comme je l'ai déjà mentionné, une récupération de la grande tradition révolutionnaire française. D'où le titre d'un éditorial du Combat de 44: "La France perdue et retrouvée". Cette France "perdue et retrouvée", c'est la France des révolutions: "...ce qui a fondé la grandeur de notre pays, ce sont précisément ces révolutions successives que les "bien pensants" condamnaient". De là vient l'importance de l'Insurrection de 1944: elle aurait récupéré ce qu'il y avait de mieux dans la France: la bonne tradition des révolutions. Août 1944 aurait repris le fil conducteur qui, pendant les sombres moments de l'Occupation, semblait rompu: "Les insurgés d'aujourd'hui retrouvent la tradition française, et cette passion de la liberté qui, comme l'amour, peut seule conduire d'un pas alerte jusqu'aux suprêmes sacrifices" (Note 3).

C'est précisément le "charme" de l'expérience révolutionaire française, pour utiliser l'expression d'Engels, qui joue un rôle de premier plan pendant la période de 1944. Cette période de "l'illusion lyrique", ou de la "révolution imaginaire", ainsi définie par une contemporaine, Dominique Desanti, dans son autobiographie politique, fut aissi, encore selon cet auteur, "l'époque de la persistance du vocabulaire révolutionnaire" (Les Staliniens. Une expérience politique) (Note 4).

Emporté par cette conjoncture de radicalisation politique, Sartre a pu développer le style enflammé déjà annoncé par exemple dans ses essais de 1938 sur Faulkner et sur John dos Passos. Ce discours pamphlétaire, cette prose vibrante bien au goût de l'époque, était 
l'expression d'une période où le système établi semblait s'écrouler. Le mot d'ordre pour une "politique radicale" (l'expression est de Sartre)-gauchisme soutenu par l'enthousiasme révolutionnaire de l'époque-deviendra la marque déposée du projet politique sartrien. Cette "hardiesse du langage" (pour employer une expression par laquelle Marx a désigné un certain type de langage propre aux moments révolutionaires), traduisait parfaitement l'esprit d'une époque dont le dénominateur commun était le désir de changements radicaux.

Mais si le projet sartrien traduit parfaitement l'esprit d'une époque, il faudrait comprendre quelle est la forme particulière que cette traduction du Zeitgeist acquiert dans l'oeuvre du philosophe.

Dans certains articles publiès par Sartre en 44 dans Les Lettres françaises (clandestines) on peut déceler l'épanouissement de quelques idées qui traverseront l'oeuvre sartrienne tout entière. Par exemple, dans l'article "La littérature, cette liberté" (avril 1944) Sartre, formulant un thème qui annonce "Qu'est-ce que la littérature?", proclame un lien intime entre littérature et politique: "...écrire, c'est réclamer la liberté pour tous les hommes (...). Tout écrivain conscient de son métier, trouve dans son activité littéraire elle-même, un devoir politique". Et dans l'article intitulé "Un film pour l'après-guerre" (avril 1944), on voit s'esquisser, entre les lignes, le grand projet philosophico-littéraire-politique qui dirigera la pensée sartrienne: la quête du social, ou mieux de l'individu dans le social; bref, la quête de la totalité. La tâche qu'il propose pour le cinéma de l'après - guerre - rendre compte de la situation historique et politique de la France occupée, réfléchir sur les problèmes de son époque - c'est exactement ce que lui-même essayera d'entreprendre désormais. Son oeuvre est, comme le grand cinéma qu'il propose, le témoignage de son époque. "C'est une grande fresque sociale qu'il aura à peindre" - dit Sartre dans ce journal de la Résistance. Cette peinture sociale, ce portrait d'une époque que Sartre avait déjà exalté chez Dos Passos, c'est aussi son propre but.

Mais c'est, sans aucun doute, dans la série de reportages sur la Libération de Paris que Sartre publie dans Combat, au fur et à mesure des événements, que l'on voit se dessiner quelques-uns des concepts sartriens fondamentaux (particulièrement le concept de révolution).

Dans ces sept articles de la série intitulée "Un Promeneur dans Paris insurgé", Sartre se présente comme un reporter qui fait le récit de ce qu'il voit: "Je ne raconte que ce que j'ai vu" - voilà la toute première phrase de cette série d'articles. Mais, bien sûr, ce regard jeté par Sartre sur les rues d'un Paris insurgé n'est pas neutre. Il n'est pas fortuit ni naif non plus. "Raconter quelque chose-comme le dit Adorno au sujet du roman contemporain - c'est avoir quelque chose de particulier a dire". ("La Situation d u narrateur dans le roman contemporain").

Or, une question s'impose donc tout de suite: qu'est-ce que le récit sartrien de 44 veut dire de particulier? Ce récit révèle que Sartre, cet écrivain impregné de phénoménologie allemande et qui connaissait déjà un peu Hegel et Marx, venait d'apprendre à lire l'histoire 
à la lumière de l'insurrection parisienne. Sous les coups de feu des barricades de 44 , une philosophie de la révolution vient d'éclore.

C'est à ce moment-là que, à mon avis, se pose pour Sartre le problème (développé dans la Critique de la raison dialectique-dorénavant CRD) de la réorganisation temporelle d'une expérience historique. Comment recréer, par la mémoire, presque à la manière d'un roman historique, l'histoire vécue? Voilà la question théorique sous-jacente aux récits de Sartre. Cette question renvoie directement au "problème de la temporalisation" étudié dans la CRD lors de l'analyse sur la Révolution Française. Et, de son côte, ce problème conduit à la catégorie de point de vue, c'est-à-dire la réorganisation temporelle ne peut être faite qu'à partir du point de vue d'un certain agent historique. Bref, on ne peut pas lire l'histoire sans prendre le parti d'un certain sujet historique (Note 5). La narration faite par Sartre des journées de 44 prend, bien sûr, le parti des insurgés - elle est l'incarnation même du point de vue du Résistant.

De la même manière que dans l'important essai écrit un an plus tard, "La Libération de Paris: une semaine d'Apocalypse" (essai reproduit intégralement dans Les Ecrits de Sartre, Gallimard, 1970), Sartre décrit, ou plutôt narre, l'Insurrection parisienne comme une "fête tragique et mortelle", une "solennité tragique", un "moment d'ivresse et de joie".

Qu'on se souvienne que postérieurement Sartre, décrivant ces moments "exceptionnels" où l'histoire est "révélée", utilise encore un langage qui suggère une approximation entre histoire et théâtre. L'histoire est conçue comme un spectacle, comme une scène, une représentation. Le moment d'une explosion révolutionnaire - dit-il par exemple dans "Les Communistes et la paix" - c'est une "cérémonie" dont le but est de "donner aux masses une représentation tragique de leurs aspirations profondes, un peu comme, selon Nietzsche, la représentation "figurée" dans la tragédie grecque reflète les plus profonds instincts du choeur" (p. 112-3). L'instant insurrectionnel, c'est ainsi le "théâtre dans la rue" que souhaitait Artaud" (p. 153). Cette relation entre histoire et tragédie est partie intégrante de toute la reconstitution faite par Sartre de l'histoire de la France. Le mouvement historique est décrit encore dans "Les communistes et la Paix" de la manière suivante: "L'histoire s'avance masquée: quand elle se découvre, elle marque les acteurs et les témoins pour toujours" (p. 274). En ce sens, par exemple, 1848 et 1871 sont conçus comme deux moments tragiques de l'histoire où la bourgeoisie, "mise à nu", voulait "crever tous les yeux du prolétari at" (p. 275).

A mon avis, cette conception "théatrale" de l'histoire, cette vision de l'histoire et de la révolution comme spectacle, pourrait être enracinée dans le récit sartrien, pris sur le vif, de l'Insurrection de 44. A l'origine de sa conception tragique du processus historique se trouve sans doute ce témoignage d'un moment politique décisif: "J'ai assisté à une fête tragique et mortelle". Sa conception tragique de l'histoire comme terreur sera, je le crois bien, l'expression philosophique de ce témoignage politique.

Le thème de la terreur est déjà à l'horizon implicite du récit sartrien de 44. Le philosophe essaye de décrire le mouvement de passage de ce qu'il appelle la "joie généreuse" d'une 
Révolution à la peur, aux "basses vengeances", bref, à la Terreur. Dans cette description on peut entrevoir l'esquisse des réflexions sartriennes postérieures sur la Terreur.

De la même façon que dans les éditoriaux du Combat, auxquels j'ai déjà fait référence, l'Insurrection parisienne de 44 figure aussi dans les articles de Sartre non pas comme quelque chose d'entièrement nouveau, mais plutôt comme une sorte de restauration ou de renaissance de la grande tradition révolutionnaire française-une récupération des journées révolutionnaires de 1789 et de 1848. "La rue-dit Sartre déjà dans le premier article de la série-est devenue, comme en 89 , comme en 48 , le théâtre des grands mouvements collectifs et de la vie sociale (Note 6). Dans cette perspective, 1944 aurait rétabli le lien rompu du processus révolutionnaire, retrouvant ainsi le temps perdu des révolutions.

Il semble donc que cette exaltation de 1944 comme une reprise de l'ère des grandes révolutions fasse partie du Zeitgeist. Comme le dit Adorno (dans son essai "La naïveté épique"), "tout récit épique contient un élément anachronique. "Le récit épique de Sartre, en redonnant vie au passé révolutionnaire de la France, cherche délibérément à confondre $1789,1830,1848,1871$ et 1944 . Il est difficile de ne pas nous rappeler ici le célèbre passage de Marx dans le 18 Brumaire: "C'est précisément quand ils semblent occupés à se transformer, eux et les choses, à créer quelque chose de tout à fait nouveau, c'est justement à ces époques de crise révolutionnaire que les hommes appellent craintivement les esprits du passé à leur rescousse". Dans le cas de 1944, dans cette conjoncture vécue exactement comme un moment de "crise révolutionnaire". c'est l'esprit du passé révolutionnaire de la France qui est invoqué pour illuminer la scène politique présente.

Cette recherche du temps perdu des révolutions fait de la Révolution Française le grand "modèle" inspirateur des analyses de Sartre (Note 7). Il pense toujours la Résistance sous l'angle de la Révolution Française. Mais, en même temps, le contraire est également vrai: c'est la mémoire nostalgique de la Résistance-conçue comme un instant privilégié de liberté et de négation où s'est réalisée la soudaine révélation de l'histoire-qui ira peupler les réflexions de Sartre sur l'insurrection révolutionnaire de 1789 et même sur celle de 1917. Il y a donc dans les analyses sartriennes une quasi identification entre Résistance et Révolution (Note 8).

Dans "Qu'est-ce qu'un Collaborateur?" - texte écrit juste à la fin de la guerre (août 1945) - Sartre définit la grande tâche politique du moment de la manière suivante: "Il faut profiter de notre victoire" - "la Résistance qui a fini par triompher" - pour réaliser une "révolution nouvelle" capable d"achever le travail que la Révolution de 89 a commencé". Voilà donc ce qui signifie pour Sartre le mot d'ordre "De la Résistance à la Révolution". L'Insurrection de 1944 devrait aboutir à une "révolution nouvelle" qui puisse réaliser vraiment "ce qu'on a tenté en 1789, en 1830, en 1848, en 1871 et qui a toujours été suivi d'une contre-révolution."

C'est pendant ce moment d'enthousiasme politique que Sartre, malgré ses critiques postérieures, va adopter quelques idées de l'Ecole Jacobine de la Sorbonne sur la 
Révolution Française. Par exemple, l'idée de 89 comme une révolution inachevée, une révolution qui ne s'est pas tout à fait réalisée (Note 9). Cette idée présuppose, bien sûr, une lecture du passé à partir du présent, plus précisément à partir du point de vue d'une révolution socialiste moderne. Cela nous renvoie encore une fois à la catégorie de point de vue à laquelle j'ai déjà fait allusion. Dans "Matérialisme et Révolution" (1946), Sartre dit explicitement qu'il "adopte" la définition de Révolution d'Albert Mathiez: "Nous adopterons la définition a posteriori qu'un historien, A. Mathiez, donne de la révolution: il y a révolution, selon lui, lorsque le changement des institutions s'accompagne d'une modification profonde dans le régime de la propriété" (p. 176). De toute façon, il y avait dans l'après-guerre français une hégémonie des idées de cette Ecole de la Sorbonne sur la Révolution Française (hégémonie exercée surtout par Georges Lefebvre, disciple d'Albert Mathiez).

La Résistance, et plus particulièrement l'insurrection de 44 , a donc posé de manière décisive pour Sartre le problème de la Révolution. Le probléme de la Révolution et le problème de la liberté, il faut ajouter (ce n'est pas par hasard qu'il conçoit la Révolution sous l'angle de la liberté). Célébrée comme un moment privilégié de liberté et de totalité où les rapports individu-histoire sont devenus transparents, la Résistance représente pour Sartre le vrai idéal d'action politique. "La Résistance-dit-il dans "La République du silence" (1944) - fut une démocratie véritable (...). Dans l'ombre et dans le sang, la plus forte des Républiques s'est constituée. Chacun de ses citoyens (...) en se choisissant lui-même dans sa liberté, choisissait la liberté de tous. Cette république sans institutions, sans armée, sans police, il fallait que chaque Français la conquière et l'affirme à chaque instant contre le nazisme". C'est de lagénéralisation théorique de ce que Lukacs a appelé "nostalgie de la simplicité poétique du temps de la Résistance" que vient, à mon avis, la conception sartrienne romantique et hérö̈que de la Révolution.

Malgré toutes ses déterminations matérielles, la Révolution signifie pour Sartre un "acte libre". Elle est le seul chemin qui puisse conduire à la libération effective de l'humanité. Et pourtant, il est bien vrai que la Révolution a aussi nécessairement une composante de violence et de Terreur: "...comme la liberté opprimée veut se libérer par la force-écrit Sartre dans "Matérialisme et Révolution" - , l'attitude révolutionnaire exige une théorie de la violence comme réplique à l'oppression (Note 10). Mais en même temps, selon Sartre, "la pensée révolutionnaire est humaniste (...). La plus sanglante des révolutions comporte-t-elle malgré tout des ralliements".

Or on pourrait se demander comment concilier ces deux idées à première vue contradictoires de révolution, c'est-à-dire révolution comme violence et terreur, et révolution humaniste et libertaire. La réponse de Sartre, développée dans la C.R.D., est actuelle et polémique (si l'on pense à l'historiographie française plus récente, particulièrement $F$. Furet et même Claude Lefort). D'après lui seulement les auteurs conservateurs, ou même "réactionnaires", opposent liberté et terreur dans un processus révolutionnaire: "Le comportement du révolutionnaire, au 14 juillet comme au 10 août, est en apparence contradictoire: non seulement il se bat pour la liberté (...) mais il réalise en 
lui la liberté souveraine comme unité et ubiquité; pourtant, dans le même temps, il fait violence à l'ennemi (...) et il use de violence perpétuelle pour se réorganiser, allant jusqu'à massacrer certains de ses propres membres. En fait il n'y a pas de contradiction (...). La liberté comme souveraineté de la praxis individuelle n'est pas violence: elle est simple réorganisation dialectique de l'environnement (...). Il n'y a d'autre contradiction que dialectique dans ces caractères si souvent opposés - par les auteurs réactionnaires - Espoir et Terreur, liberté souveraine en chacun et violence exercée contre l'Autre, hors du groupe et en lui". (C.R.D., p. 428-9). Et un peu plus loin, reprenant implicitement quelques thèses soutenues par Merleau-Ponty dans Humanisme et Terreur, Sartre justifie la violence révolutionnaire de la manière suivante: "La Terreur, c'est la violence de la liberté commune contre la nécessité" (p. 448). Dans le tome ll inachevé de la C.R.D., ce recours à Humanisme et Terreur devient explicite: "A un certain degré d'urgence, dans le climat de la fraternité-terreur, toute opposition, comme l'a dit Merleau-Ponty, est trahison" (p. 72). Le lien intime entre Vertu et Terreur, tel que les Jacobins l'ont établi, est aussi sọuligné par Sartre dans son manuscrit inédit Joseph LeBon.

Ce projet de réunir dialectiquement humanisme, liberté, violence et terreur est constitutif de la pensée sartrienne de la Révolution. Ce qui nous renvoie au problème plus ample - qui a toujours hanté Sartre-des rapports entre subjectivité et objectivité, ou liberté et nécessité, dans l'histoire. Dans le manuscrit inédit Joseph LeBon, ayant sans doute à l'horizon implicite le célèbre passage de Marx du 18 Brumaire - "Les hommes font leur propre histoire, mais (...) en même temps ils sont faits par l'histoire" - , Sartre écrit: "Marat est fait par le XVIIIe. Mais il fait la révolution (lui et des millions d'autres)".

De la même façon que le projet théorique de Sartre incarne l'idéal d'une synthèse entre objectivité et subjectivité dans l'histoire, son projet politique incarne aussi l'idéal (soutenu par la Résistance) d'une synthèse entre révolution et liberté, socialisme et liberté. "Le socialisme - dit-il dans l'interview accordée à Michel Contat en 1975-, c'est la liberté se prenant elle-même pour fin" (Situations X). D'où le nom de son groupe d'intellectuels résistants fondé en 1941: "Socialisme et Liberté". "C'était ce lien entre socialisme et liberté - confirme Sartre dans un entretien avec Simone de Beauvoir - qui représentait ma tendance politique". (La Cérémonie des adieux). Que l'on se souvienne qu'à l'époque du R.D.R. (Rassemblement Démocratique Révolutionnaire) - groupe politique créé en 1948 par une gauche indépendante du P.C. - le but de Sartre était, comme il le dit dans les Entretiens sur la Politique, d'unir les revendications révolutionnaires à l'idée de liberté." Dans des notes inédites, citées par Simone de Beauvoir (La Force des Choses), Sartre affirme que sa réflexion politique à l'époque du R.D.R. venait d'une "idée inspirée par la Résistance". C'etait de cette manière que le philosophe pensait mettre en pratique le grand mot d'ordre qui a alimenté les rêves de toute une génération en 44: pour une Révolution qui réalise vraiment la liberté (Note 11).

Dans le sillage du jeune Marx, Sartre veut, d'après ce qu'il écrit dans "Matérialisme et Révolution", lier "les intérêts de l'esprit" et le prolétariat. Son projet de "lutter pour la révolution" est déjà présenté en 1944 dans "A Propos de l'Existentialisme: Mise au Point": "La lutte des classes est un fait, j'y souscris entièrement: mais (...) elle se situe sur le plan 
de la liberté". Démocratie? Oui, dit-il dans les Entretiens sur la Politique, mais "seulement à travers la liquidation de sa structure sociale capitaliste, c'est-à-dire par ce qu'on appelle une révolution". Seulement une transformation révolutionnaire pourrait donc établir la vraie liberté (Note 12).

Ayant mis le thème de la Révolution à l'ordre du jour, permettant ainsi aux échos des révolutions française et russe de se propager chez les intellectuels de l'époque, la Résistance amène Sartre à réfléchir sur ce qu'il appelle, dans ses reportages de 44, "naissance de l'esprit insurrectionnel". D'où le titre du deuxième article de la série "Un Promeneur dans un Paris insurgé": "Naissance d'une Insurrection". C'est dans la tentative pour comprendre la nature de l'Insurrection de 44 que Sartre, à mon avis, commence à formuler le problème de la formation des groupes (thème de la C.R.D.). Le concept sartrien de "groupe en fusion" a été peut-être forgé à chaud pendant les événements de cette "semaine d'Apocalypse". Sartre lui-même a assisté à la formation d'un groupe en fusion et a essayé de décrire ses structures. C'est de cette description (politique) que, je le crois bien, surgit pour l'auteur le problème (théorique) de la formation de groupes. Faisant référence justement aux "groupes qui se forment", Sartre écrit dans le troisième article de la série, "Colère d'une ville", la chose suivante: "Toute la matinée, c'est la colère qui souffle sur la ville. Cette foule a enfin décidé de prendre son destin entre ses propres mains. Vers 11 heures, on voit apparaître les premières barricades. Le chemin qui mène de la docilité douloureuse à l'insurrection est enfin parcouru. A partir de ce moment, il n'y aura plus que des combattants". C'est probablement dans cette description du passage d'un état "de paix à la guerre", pour utiliser encore l'expression de Sartre dans ce troisième article, que se trouve l'origine de ce que l'auteur appelle, dans la C.R.D., le "passage d'un monde ossifié et refroidi à une Apocalypse" (p. 410). L'origine politique du thème sartrien de l'Apocalypse (outre son origine littéraire qui vient, comme le dit Sartre lui-même, de L'Espoir de Malraux) se trouverait donc dans ce récit, fait au fur et à mesure des événements, d'une "manifestation collective" guidée par "l'esprit de solidarité" (les expressions sont de Sartre) (Note 13).

F. Jameson écrit, dans son livre Marxism and Form (Princeton University Press, 1971), que "le concept sartrien du groupe vise une petite unité du type guérilla et tend à rendre impossible l'idée d'une classe sociale comme agent de l'histoire". Sans aucun doute, mais pas seulement... En dépit de son grand intérêt, l'analyse de Jameson laisse de côté l'origine historique de ce concept sartrien de groupe. Ce concept, comme j'essaie de l'indiquer, possède un substrat historique réel: l'Insurrection de 44. Sartre lui-même, dans ces reportages pour Combat (auxquelles Jameson ne fait pas référence), emploie le mot de "guérilla" pour désigner le type d'activité des Résistants. Ajoutons que la presse de l'époque appelait l'activité des Résistants "guérilla". L'idée d'une classe sociale comme sujet de l'histoire ne se soutient pas peut-être à plusieurs moments de l'analyse sartrienne parce que le philosophe pense à une "race", selon son expression, des Français unis. Par exemple, dans le sixième article de la série "Un Promeneur dans Paris insurgé" ("La Délivrance est à nos portes"), Sartre, avec "le coeur en fête" et dans une extase d'enthousiasme face à la foule qui "hurle de joie", est amené à parler d'une "seule race des Français libres" (c'est moi 
qui souligne). A ce moment-là, le grand mythe de la Résistance-l'union nationale - engendre la dilution des classes sociales à l'intérieur d'un tout amorphe: la race, la nationalité. C'est l'expérience de la "fraternité virile", de la lutte en commun contre l'ennemi en commun qui se trouve toujours à l'horizon des réflexions sartriennes. Et cela Jameson ne nous le montre point. Même les relations sociales pendant la période de la Révolution Française sont décrites par Sartre, dans son manuscrit inédit Joseph LeBon, comme "amitié révolutionnaire". Ce n'est pas par hasard que dans "Matérialisme et Révolution" l'auteur se propose de chercher une "philosophie de la révolution" qui soit "la philosophie de l'homme en général." Or, on pourrait se demander quelle est la place réservée à la lutte des classes (à laquelle Sartre, déjà en 44, disait "souscrire") dans cette "philosophie de l'homme en général"?

Il semble donc qu'à l'origine de l'idée sartrienne de la Révolution comme un instant d'Apocalypse dans l'histoire se trouve l'expérience politique vécue par le philosophe en 44. On voit mieux maintenant en quel sens on pourrait affirmer qu'il y a une expérience historique réelle à l'intérieur du mouvement spéculatif des concepts philosophiques de Sartre. Ces concepts ont été engendrés sur le sol historique d'une France qui luttait pour sa Libération. Comme Hegel l'avait déjà fait à l'égard de 89 (selon l'interprétation d'Habermas dans "Hegel critique de la Révolution Française"), Sartre a voulu "légitimer dans le concept une révolution de la réalité".

Les analyses sartriennes sur la Révolution ont toujours comme toile de fond l'Insurrection parisienne de 44. Par exemple, dans l'article "La Libération de Paris: une semaine d'Apocalypse", Sartre, faisant une comparaison entre la prise de la Bastille et l'Insurrection d'août 44, essaye de montrer que ces deux événements ont en commun "l'air de fête", "l'exercice de l'A pocalypse", "l'explosion de la liberté", "la rupture de l'ordre établi". Voici, dans les mots de l'auteur, cette célébration de 44: "En ce mois d'août, les combattants qu'on rencontrait dans les rues (...) s'enivraient, en face d'un ennemi bardé de fer, de sentir la liberté (...). Et l'on ne pouvait s'empêcher de penser à ce que Malraux nomme, dans L'Espoir, l'exercice de l'Apocalypse. Oui, c'était le triomphe de l'Apocalypse, cette Apocalypse toujours vaincue par les forces de l'ordre et qui, pour une fois, était victorieuse. L'Apocalypse: c'est-à-dire une organisation spontanée des forces révolutionnaires. Tout Paris a senti, dans cette semaine d'août, que les chances de l'homme étaient encore intactes, (...) ces quelques jours ont suffi pour prouver la puissance de la liberté." Ce texte vient donc confirmer l'origine du thème de l'Apocalypse chez Sartre: il s'agit vraiment d'une reprise du roman de Malraux sur la guerre civile à la lumière de l'Insurrection parisienne de 44. Dans la C.R.D., la description du moment majeur de la Révolution Française correspond exactement à la description que l'auteur avait déjà faite de l'instant insurrectionnel de 44: "...ce que Malraux a appelé, dans L'Espoir, l'Apocalypse c'est-à-dire la dissolution de la série dans le groupe en fusion" (p. 391). Et après il ajoute: "C'est la France comme Apocalypse qu'ils découvrent à travers la prise de la Bastille" (p. 410-1).

Mais si d'un côté il est bien vrai que l'idée sartrienne de la Révolution comme Apocalypse renvoie à Malraux, de l'autre côté il faudrait ajouter que la source de cette idée remonte à une époque encore beaucoup plus lointaine: elle date du XVIIIe siècle. Ce sont les 
révolutionnaires eux-mêmes de cette époque qui, d'après les analyses de Milton Nascimento (dans son livre Opinião Pública e Revolução, São Paulo, EDUSP, 1989), ont déjà une conception apocalyptique de la révolution qu'ils sont en train de faire. L'enthousiasme et la passion révolutionnaires déclenchaient dans le coeur de chaque militant la sensation de "sortir des cendres", de renaître, d'aller "de la mort vers la vie" - une sorte de résurrection glorieuse. C'est parce que ces protagonistes de 1789 comprenaient très bien le caractère tout à fait extraordinaire du moment qu'ils étaient en train de vivre que la Révolution peut s'exalter à elle-même d'une manière si solenelle (comme d'ailleurs Michelet, de qui Sartre était un grand lecteur, l'a vivement montré). Dans le langage de l'époque la Révolution fut saluée, selon Starobinski (1789-Les Emblèmes de la raison. Champs-Flammarion, 1979, cité par Nascimento), comme culte, sermon, cérémonie, fête, célébration. Rappelons-nous que Sartre utilise exactement le même vocabulaire pour désigner les moments d'explosion révolutionnaire.

Toutefois, cette reprise sartrienne de l'esprit du XVIIIe siècle est, il me semble, très particulière. Il s'agit, à mon avis, d'un XVIIIe siècle tel qu'il a été vu, ou plutôt lu, par un auteur du XIXe siècle, Victor Hugo. D'une manière très vivante Hugo reprend au siècle suivant cette conception apocalyptique de la Révolution Française professée par les révolutionnaires de l'époque eux-mêmes. "La révolution française-lit-on dans Les Misérables (ce livre qui, selon Hugo, était contenu en germe dans son poème intitulé Mélancholia) - est le plus puissant pas du genre humain depuis l'avènement du Christ. Incomplète, soit, mais sublime. (...) Elle a adouci les esprits; elle a calmé, apaisé, éclairé; elle a fait couler sur la terre des flots de civilisation. Elle a étébonne. La révolution française, c'est le sacré de l'humanité" (V. I, p. 42-3). Ou encore: "la révolution, prise dans son ensemble, est une immense affirmation humaine. (...) De ses coups les plus terribles, il sort une caresse pour le genre humain" (V. I, p. 46). A travers les paroles d'un personnage qui a été membre de la Convention, Hugo exalte encore la Révolution Française comme "fraternité", "concorde", "fête", "aurore", "sacré".

On pourrait maintenant élargir quelques considérations déjà présentées. Oui, l'idée sartrienne de révolution comme Apocalypse est sans aucun doute une reprise du roman de Malraux sur la guerre civile à la lumière de l'Insurrection parisienne de 44. Mais plus que cela, c'est un appel héroïque-d'où le récit épique -à l'esprit de 89 . Et il faudrait encore ajouter: c'est, semble-t-il, l'esprit du XVIIIe siècle incarné chez un auteur du XIXe siècle, Victor Hugo, qui illumine la reconstitution sartrienne des journées révolutionnaires de 4. Bien que Sartre ne fasse pas allusion à Hugo, c'est probablement ce dernier (outre Malraux) qui est à l'horizon de sa conception apocalyptique de la Révolution.

Il convient de reprendre ici un passage fondamental des Cahiers pour une Morale (1947-1948) où l'héritage de cette conception apocalyptique de la Révolution se fait sentir très nettement: "Ainsi la liberté fait exploser perpétuellement l'idéologie, la mythologie et les rites antérieurs: elle réalise la libération par la conduite et l'idée neuve. C'est le moment de l'Apocalypse (c'est aussi le moment de la fête). (...) Le moment humain, le moment de la morale est celui de l'Apocalypse, c'est-à-dire de la libération de soi-même et d'autrui dans une reconnaissance réciproque. C'est aussi le plus souvent-paradoxalement-le 
moment de la violence. (...) Fête, apocalypse, Révolution permanente, générosité, création, voilà le moment de l'homme. (...) Il ne peut y avoir de liberté que dans la libération" (p. 429-30).

L'Apocalypse - cet instant de "vraie dialectique historique", ou de "récupération de l'absolu" (p. 487), pour reprendre encore des expressions des Cahiers pour une morale-, c'est le moment privilégié de communion entre le particulier et l'universal dans l'histoire. C'est le moment de la totalité historique. "Dans l'Apocalypse-écrit Sartre dans la C.R.D. - (...) l'unité synthétique est toujours ici, où, si l'on préfère, en chaque lieu de la ville, à chaque moment, dans chaque processus partiel, la partie se joue tout entière et le mouvement de la ville y trouve son achèvement et sa signification" (p. 391).

Pierre Victor affirme (dans son article "Apocalypse", publié dans Obliques, 1978) que lorsque Sartre a découvert l'Apocalypse" (et il l'a "découverte", comme j'essaie de le montrer, en 44) il a découvert la Révolution. Oui, Sartre lui-même, par exemple dans le Tome 11 de la C.R.D. (p. 165) identifie Révolution et Apocalypse (Note 14). Mais on pourrait ajouter: il a découvert en même temps l'histoire comme le chemin de la Révolution. Ou mieux, pour employer ses mots dans l'interview accordée à Michel Contat, la révolution comme "le moment décisif de l'histoire".

"Lorsque la foule de 1789 envahit la Bastille - affirme Sartre ("La Libération de Paris: une semaine d'Apocalypse) - elle ignorait la signification et les conséquences de son geste; c'est a près coup, peu à peu, qu'elle en a pris conscience et qu'elle l'a élevé à la hauteur d'un symbole". Or, on pourrait dire la même chose au sujet de Sartre lui-même: il a élevé l'expérience révolutionnaire vécue en 44 "à la hauteur d'un symbole". Ce moment-là - décrit dans sa série de reportages comme un "carnaval de guerre", une "grande fête presque religieuse" - sera cristallisé théoriquement par le philosophe et converti en fétiche, en mythe. La fascination produite par cette "cérémonie extraordinaire", pour cet instant où, face à "toute une ville aux barricades", "l'espoir monte dans tous les coeurs", conduit Sartre à ce que F. James a appelé, très judicieusement, "survalorisation du moment de la formation du groupe" et "mystique de l'Apocalypse".

"Je ne raconte que ce que j'ai vu" - avait dit Sartre, comme je l'ai déjà fait remarquer, dans le premier article de ses reportages. Et, à une autre occasion, il avait déjà annoncé: "ne plus faire d'autres récits que ceux d'événements de ma propre vie". On pourrait ajouter que son oeuvre, particulièrement sa pensée sur l'histoire et la révolution, c'est essentiellement le récit, la narration, de l'instant "Apocalyptique" de 44. Il n'est pas fortuit que le témoignage d'un autre moment révolutionnaire, celui de 1848 , mis en forme littéraire par Flaubert, exercera une énorme influence sur Sartre. D'ailleurs, le thème de la Révolution semble être toujours au fond des analyses sartriennes sur Flaubert. "L'Education sentimentale a la valeur d'un manifeste", disait Sartre, bien avant L'Idiot de la famille, dans "L'Homme ligoté" (1945). Déjà dans Les Carnets de la drôle de guerre, Sartre, après avoir critiqué radicalement L'Education sentimentale, affirme que la vraie 
"importance de Flaubert", c'est que "son style est de transition", c'est-à-dire le résultat des "mouvements sociaux de 48" (p. 130). Mais l'oeuvre sartrienne n'est-elle pas elle-même le résultat des mouvement sociauxqui ont aboutià une période de conflits politiques sanglants et radicaux? Et le témoignage de Sartre aura aussi une forme presque littéraire. Il raconte l'Insurrection de 44 comme une histoire dont les personnages sont les Allemands, la foule parisienne, les Résistants, les Insurgés. Dans le" style de Michelet, la reconstitution sartrienne d'un moment révolutionnaire acquiert une forme quasi romanesque. Le grand modèle de Sartre est toujours littéraire (Note 15). "J'aurais rêvé, disait-il déjà en 1938, de n'exprimer mes idées que dans une forme belle - je veux dire dans l'oeuvre d'art, roman ou nouvelle." L'oeuvre sartrienne pourrait ainsi être considérée une sorte de "roman philosophique" qui exprime une expérience politique fondamentale. C'est comme s'il y avait sous-jacente à la pensée de Sartre une théorie du roman liée à une théorie de la Révolution.

Comme les analyses de Jameson l'ont vivement indiqué, la réflexion sartrienne sur la Révolution cherche à "recréer synthétiquement dans la pensée", à la manière d'un romancier historique (d'où l'aspect fictionnel du récit), l'expérience historique concrète. Cette réorganisation temporelle de la situation historique réelle, cette remémoration du temps perdu par le récit historique, entraîne le lecteur à répéter le même mouvement du lecteur d'un roman historique, c'est-à-dire qu'il devient complice de quelques personnages. Bref, il prend parti.

Il reste à souligner que ce lien entre roman et révolution, cette narration épique qui exprime un temps révolutionnaire, est elle-même le résultat d'un moment historique privilégié où était donnée la possibilité de dévoiler le social comme totalité. "La particularité du moment révolutionnaire-écrit Jameson (dans Marxism and Form) - c'est qu'en lui l'histoire prend la forme d'événements narrables, elle se révèle avec une continuité, avec un début, un milieu et une fin". Cela nous renvoie au problème (déjà étudié par Lukacs dans Histoire et conscience de classe) des rapports entre révolution et totalité. C'est le processus révolutionnaire lui-même - ce processus totalisant et totalisateur qui engage la collectivité tout entière - qui permet la narration épique.

Nous revenons ainsi à notre point de départ. La forme narrative de Sartre serait l'expression théorique d'un moment de totalisation historique, c'est-à-dire d'un moment, pour reprendre la description faite dans la CRD du cas du 14 juillet, d'homogénéité d'une ville en fusion" où "la réalité constituée n'existait plus" et où "la violence du danger et de la passion surmontait les hétérogénéités sociales" (p. 395). L'idéal sartrien de synthèse entre le singulier (le particulier) et l'universel trouve dans la "semaine d'Apocalypse" de 44, dans cet instant où chaque individu est inexorablement emporté par le mouvement totalisateur de l'histoire, sa réalisation effective. Sartre lui-même, pris par ce processus total de transformation historique, et essayant de répondre "au défi que lui lance la Révolution", érige la Révolution elle-même en "principe de sa philosophie" (comme d'ailleurs Hegel, selon la lecture d'Habermas mentionnée plus haut, l'avait aussi déjà fait à l'égard de la Révolution Française). Sartre pensera désormais l'histoire dans la perspective de la Révolution. "Je montre l'organisation à partir de l'Apocalypse - dit-il dans la CRD - et l'on pourrait faire le contraire" (p. 433). C'est pendant ces "jours mémorables" de passion 
politique que Sartre, reprenant l'esprit du passé révolutionnaire de la France, dévoile la situation historique présente.

Que conclure? Les réflexions sartriennes sur l'Histoire et sur la Révolution seraient, comme j'ai essayé de le montrer, une sorte de dramatisation, de mise en scène, ou plutôt de remémoration de la situation politique vécue durant la guerre, l'Occupation et surtout durant l'Insurrection d'août 1944. Dans cette perspective, je trouve très appropriée la formule avec laquelle Michel Contat (dans la préface à la Pléiade) définit la philosophie sartrienne: "philosophie narrative". Oui. Et l'on pourrait ajouter: il s'agit essentiellement d'une philosophie de la révolution (de là la place centrale du thème de l'Apocalypse) qui narre, de manière épique (à la façon d'une épopée moderne), l'expérience politique vécue dans un de ces moments privilégiés où "l'histoire en suspens", d'après l'expression de Merleau-Ponty, rend possible l'émergence d'une "nouvelle manière de voir", comme le voulait Sartre en 1945 (dans "L’Homme ligoté").

"Une nouvelle manière de voir" qui, essayant de rendre compte d'une expérience historique particulière, finit curieusement par généraliser cette expérience et, ce faisant, récupère subrepticement de vieilles formes de spéculation. La situation politique concrète, généralisée, devient presque une catégorie spéculative. Si d'un côté la philosophie sartrienne incarne un fait historique réel (elle est le témoignage vivant d'une époque de "haute température historique", pour utiliser l'expression de Jaurès), de l'autre côté, ce fait historique figure plusieurs fois dans les textes de l'auteur comme un "fait métaphysique". La généralisation théorique (et rhétorique) de l'expérience politique de la Résistance risque ainsi de déboucher sur une métaphysique de la Révolution (qui surpasse les problèmes théoriques et politiques d'une révolution historique déterminée). Il en résulte parfois la dilution, ou la volatilisation, de l'histoire réelle à l'intérieur du mouvement des formes purement spéculatives. L'oeuvre sartrienne pourrait donc être lue comme le résultat d'un amalgame de forme spéculative traditionnelle (dans le sens que l'Ecole de Francfort a donné à ce terme), d'expérience politique cruciale et de récit philosophique. Elle serait le produit de cet amalgame -à la fois philosophie et forme littéraire.

Mais malgré toutes ses difficultés, la philosophie sartrienne - cette philosophie capable de "penser à grands coups d'épée", comme Sartre lui-même définissait la pensée révolutionnaire - a su formuler avec beaucoup de sensibilité les grands problèmes théoriques et politiques de notre époque (par exemple, le problème des rapports entre socialisme et liberté, révolution et liberté-problème remis aujourd'hui à l'ordre du jour par la Perestroika en Union Soviétique et par le printemps de Pékin si affreusement écrasé).

Dans son adhérence désespérée aux problèmes du monde, Sartre est l'héritier fidèle d'Hegel pour qui "une philosophie qui faillit à sa tentative de saisir son époque en pensée (...) n'est qu'une grossière abstraction". L'actualité de cette mise en garde hegelienne se fait encore vivement sentir en ce moment où règne le vide des idéologies post-modernes. Dans cette fin de siècle si sombre les espoirs révolutionnaires ont pâli et toute une tradition fondée sur l'idée de révolution (tradition qui remonte à Hegel) a été reléguée au statut d'un chapitre de l'histoire des grandes utopies sociales. Ce n'est donc pas par hasard que la 
pensée sartrienne est elle aussi entrée en déclin. Pour le regard hautain que les idéologies post-modernes portent sur le monde l'idée de l'engagement, le propos de Sartre de comprendre son époque et son éloge de la Révolution sont devenus inșupportablement désuets - une sorte de genre désuet.

Face à l'idéologie en vigueur du refus de l'histoire et de la révolution (présente paradoxalement même chez plusieurs penseurs qui aujourd'hui fêtent le bi-centenaire de la Révolution Française), face à l'hégémonie des formules abstraites et au désintérêt prépondérant à l'égard du concret, il devient encore plus opportun de reprendre les problèmes posés par Sartre. Les tendances actuelles à se débarrasser très rapidement de ces problèmes sont peut-être des "gestes d'adieux trop pressés".

\section{NOTES}

1-Comme on le sait, la question du héros avait déjà été depassée par Hegel qui a démontré (dans son Esthétique) l'impossibilité du héros dans la société capitaliste. Selon lui, dans le capitalisme (monde prosaïque) il n'y a pas de place pour le héros. Chez Cervantes, par exemple, nous avons déjà le héros en parodie. Mais la guerre et la Résistance, reliant étroitement morale et politique, ont redonné vie aux thèmes de l'héroïsme, de l'engagement, de l'humanisme et de la liberté.

2-J'ai pu consulter ce manuscrit grâce aux efforts et à la gentillesse de Michel Contat et de Yvan Cloutier que je remercie très vivement. Ce manuscrit appartient à la Bibliothèque de l'Université d'Ottawa; un autre manuscrit faisant partie du même projet est déposé au Humanities Research Center, University of Texas, Ostin, mais je n'ai pas pu le consulter.

3-Pour bien comprendre cet esprit de 44, il ne faut pas oublier le rôle décisif que le passé révolutionnaire de la France a toujours joué dans la pensée politique. Dans La Phénoménologie de l'esprit, Hegel avait déjà salué la Révolution Française - considérée par lui comme l'expérience de la "liberté absolue" - avec ces mots d'enthousiasme: "Le ciel est descendu et transporté sur la terre". Et à la fin de sa Philosophie de l'Histoire, Hegel célèbre l'expérience révolutionnaire de 1789 dans les termes suivants: "La Révolutiom Française fut une magnifique aurore. Tous les êtres pensants célèbrent cette époque; l'enthousiasme de l'esprit ébranla le monde, et ce n'est qu'à ce moment là que l'effective réconciliation du divin avec le monde se fut accompli". C'est à partir de cette expérience révolutionnaire de 89 que le problème des rapports entre la théorie et la pratique s'est imposé à Hegel. La Révolution Française fut vraiment, comme l'a dit Marx, "l'âge classique de l'intelligence politique" ("Le roi de Prusse et la réforme sociale" -1844). D'où le bilan critique fait par Engels (dans son Introduction de 1895 aux Luttes de classes en France) de l'influence décisive que l'expérience révolutionnaire 
de la France a exercé sur sa pensée et sur celle de Marx: "...nous étions tous, quant à la façon dont nous concevions les conditions et le cours des mouvements révolutionnaires, sous le charme de l'expérience historique passée, et notamment de celle de la France".

4-Inutile de rappeler que l'expression "l'illusion lyrique", utilisée par D. Desanti, vient du titre de la première partie du livre de Malraux, L'Espoir.

5-Comme on le sait, c'est précisément cette catégorie de point de vue que François Furet et son groupe critiquent aujourd'hui dans l'historiographie jacobine de la Sorbonne.

6-D’où l'idée postériuere de Révolution comme "théâtre dans la rue".

7-Cela éloigne encore plus Sartre de la tradition du P.C.F. Aragon, par exemple, dans un poème conforme à la ligne officielle du Parti, et intitulé "Réponse aux Jacobins", répudie "La Marseillaise" comme représentante de la social-démocratie.

8-Cette identification entre Résistance et Révolution est aussi présente chez plusieurs intellectuels de l'époque - c'est le cas par exemple de Merleau-Ponty qui examine la Révolution Russe et les procès de Moscou sous l'optique de la Résistance et des procès des Collaborateurs.

9-De là le passage de Sartre que je viens de citer: "il faut achever le travail que la Révolution de 89 a commencé".

10-Rappelons-nous que pour les représentants de la tradition historiographique jacobine la révolution se fait au nom d'une violence justifiée car elle est, comme le dit Aulard, "la réponse à la violence d'un passé qui ne voulait pas mourir."

11-D'où le caractère paradoxal de cette philosophie sartrienne de la liberté et de la contingence qui, dans les années 50, a essayé de montrer la nécessité de la politique stalinienne.

12 - On voit ainsi que Sartre se trouve très loin de l'idée, proposée par Camus dans les pages de Combat, d'une voie pacifique, ou constitutionnelle, vers le socialisme. "Nous sommes démocrates et révolutionnaires", "nous sommes du côté anticapitaliste", répétait-il à l'époque du R.D.R. Ce radicalisme politique de Sartre déclenchait encore le reproche du libéral Aron. C'est précisément à cette époque-là que les divergences 
politiques entre les deux philosophes deviennent plus aigus. Pourtant, ces divergences ne nous autorisent pas à minimiser l'influence que le "relativisme historique" d'Aron a exercée sur la pensée sartrienne. Dans Les Carnets de la drôle de guerre, Sartre, bien que critiquant déjà ce qu'il appelle l'"idéalisme" d'Aron, affirme que sa découverte de l'histoire fut d'abord "philosophique", c'est-à-dire qu'elle s'est faite à travers "L'Introduction à la philosophie de l'histoire" d'Aron. Dans L'Etre et lenéant, les échos de la réflexion d'Aron sur l'histoire se font encore sentir: "Cette discussion touchant la valeur, l'ordre et la nature de notre passé, est d'ailleurs tout simplesment le choix historique en général (...) Ainsi faudrait-il une histoire humaine finie pour que tel événement, par exemple la prise de la Bastille, reçût un sens définitif" (p. 557-8). Ou alors: "... la réponse à cette question: "Quel sera, en définitive, le destin historique de Robespierre?" dépend de la réponse à cette question préalable: "L'histoire a-t-elle un sens?", c'est-à-dire "doit-elle s'achever ou seulement se terminer?" Cette question n'est pas résolue-elle est peut-être insoluble, puisque toutes les réponses qu'on y fait (...) sont elles-mêmes historiques" (p. 603).

13 - "Le caractère essentiel du groupe en fusion - écrit Sartre dans la C.R.D., -c'est la brusque résurrection de la liberté" (p. 425). Or ce passage corrobore notre hypothèse qui veut que la réflexion sur la Révolution développée dans la C.R.D. soit essentiellement une généralisation théorique de l'expérience politique de 44. C'est encore cette généralisation théorique de l'expérience politique de la Résistance qui permet à Sartre d'affirmer, comme il le fait par exemple dans son essai sur Descartes ("La Liberté cartésienne" 1945), que la liberté est l'essence de l'homme.

14-Dans "Faux savants ou faux lièvres?" (1950), Sartre présente le mouvement révolutionnaire comme un "mouvement insurrectionnel, dialectique et apocalyptique".

15-Cela explique pourquoi à partir d'une réflexion sur le roman de Faulkner, Le Bruit et la fureur ("La Temporalité chez Faulkner" 1939), s'esquissent, semble-t-il, les idées sur la temporalité développées dans L'Etre et le néant.

\section{REMERCIEMENT}

Je remercie vivement Michel Contat qui a eu la gentillesse de faire une révision technique de ce texte. Je remercie aussi beaucoup David Sehl d'avoir si amicalement relu et tapé ce texte à la machine. 
CREMA, C.D.M. - O tema da revolução dentro do pensamento de Sartre. Trans / Form/ Açãa, São Paulo, 13 : $21 \div$ 40, 1990.

RESUMO: A obra de Sartre poderia ser lida como a encarnação do espírito "patético" $e$ "heróico" da época da guerra e da Resistência. Sob o impacto das barricadas de 44, uma filosofia da revolução começa a desabrochar-ela foi forjada a quente durante esta época de "alta temperatura histórica". É da generalização teórica dessa experiência política crucial, isto é, da cristalização do "mito" da Resistência, que vem, a meu ver, a idéia sartriana de revolução.

UNITERMOS: Resistência; revolução; apocalipse; socialismo; liberdade.

\section{BIBLIOGRAFIA}

CONTAT, M. \& R YBALKA, M. Les écrits de Sartre. Paris, Gallimard, 1970.

SARTRE, J.-P. Cahiers pour une morale. Paris, Gallimard, 1983.

SARTRE, J.-P. Les carnets de la drôle de guerre. Paris, Gallimard, 1983.

SARTRE, J.-P. Critique de la raison dialectique. Paris, Gallimard, 1960.

SARTRE, J.-P. Critique de la raison dialectique. Paris, Gallimard, 1985. v.2.

SARTRE, J.-P. L'être et le néant. Paris, Gallimard, 1943.

SARTRE, J.-P. Joseph LeBon. (manuscrit inédit).

SARTRE, J.-P. La litterature, cette liberté. Les Lettres Françaises. Paris, avr., 1944. (clandestines).

SARTRE, J.-P. Merleau-Ponty vivant. Les Temps Modernes. Paris, 17 (184/5):304-76, 1961.

SARTRE, J.-P. Oeuvres romanesques. Paris, Gallimard, 1981. (Bibliothèque de la Pléiade).

SARTRE, J.-P. Un promeneur dans Paris insurgé. Combat. Paris, août, 1944.

SARTRE, J.-P. Situations I. Paris, Gallimard, 1947.

SARTRE, J.-P. Situations III. Paris, Gallimard, 1949.

SARTRE, J.-P. Situations VI. Paris, Gallimard, 1964.

SARTRE, J.-P. Situations X. Paris, Gallimard, 1976.

SARTRE, J.-P. La transcendance de l'ego. Paris, J. Vrin, 1985. 